\title{
7 Media combination, transmediation and media representation
}

\author{
Jørgen Bruhn and Beate Schirrmacher
}

In Part I, we demonstrated and exemplified different types of interactions and how technical media of display relate to basic and qualified media types. We also discussed how the functions of different media types are qualified by historical and social contexts. In Part II, we explore the intermedial combinations and different forms of transformations. We present analytical models and case studies from a variety of different qualified media types in different historical settings.

The same media product can always be approached from different perspectives. A synchronic perspective explores the interaction of different basic or qualified media types - we call this the media combination perspective. We can even explore the diachronic aspect - we call this the perspective of media transformation. Here, we can either explore the transfer of ideas or narratives across media (transmediation), or the media representation of one media type or product in another (Elleström 2014).

Discussing the media combination means being interested in the combination and integration of media types in particular media products of qualified media types. Media combination and integration do not refer to two different sorts of media products but express a different analytical focus. The different basic media types can be produced separately and combined in the production process. To the audience of a live-action film, for instance, the moving images, sound effects, music and speech can be analytically divided into basic media, but are experienced as a unity because they are deeply integrated into the sensorial modality (see Chapter 2). When we approach media combinations with the four modalities, we can see how the material and sensorial integration of basic qualified media types enables intricate combinations of different forms of meaning-making that support and interact with each other.

We can explore the combination and integration of different modes in the different modalities of one particular media product and any qualified media type. Different basic media types can share the same material interface, for instance pages and screens when we think of the combination of text and image, or sound waves when we think of the lyrics and melody in a pop song. Other qualified media types combine different material interfaces, like screens and loudspeakers in film, which provide an integrated audiovisual experience.

DOI: $10.4324 / 9781003174288-9$ 
The material space of the theatre stage allows for all kinds of basic and qualified media to be combined in a performance.

In the combination dimension, intermedial and multimodal approaches overlap. A multimodal analysis provides the tools to analyse the synchronic integration not only between but within basic media types, like text, image and organized sound, and involves an even more fine-grained analysis, understanding, for instance, how the typography, colour, layout of a text is part of the meaning-making of a text.

The broad term media transformation refers to all kinds of processes in which the form or content of one media type is reconstructed and thus transformed by another media type, for instance, a certain narrative (say, the plot of a book) or a set of ideas (say, the hypothesis of a scientific article) or the visual aesthetics of an oil painting that is reused in a commercial ad. All media transformation relies on two interrelated aspects: transmediation and media representation.

Transmediation reconstructs meaning that was previously mediated by another media type; a film adaptation, for example, may mediate the same story as a novel. When we analyse transmediations, we focus on a diachronic process and we explore the relation between a source media product and a target media product and analysing what is transferred and what is transformed. We often speak of transmediations as if they were a transfer of the media product, how a 'novel is turned into a film'. But strictly speaking, it is not the source media product that is transformed. Instead, it is the ideas, narratives and concepts previously communicated in the source media product that are reproduced or reconstructed in the target media product. Therefore, in the process of transmediation, narratives and ideas can only be transferred across media by being transformed. The analysis of transmediation investigates the interplay between medium specificity and transmediality, asking how transmedial concepts and structures of a source media product are reconstructed in the target media products in a media-specific way.

When exploring media representation, we analyse how one medium represents the characteristics of another medium, such as when a poem describes a painting not only by way of representing the image or the depicted scene but also offers a depiction of the painting as an object. Media products constantly refer to and represent other media products or media types or specific technical media of display. By representing other technical devices and basic media types, a media product like a video game also refers to the contexts and conventions of the represented media types. Therefore, media representation is sometimes discussed as an intermedial reference. By representing other media, media products almost by necessity draw on the history and content connected with the represented media. We can explore media representation in the storyworld, and we can also explore how the presentation of a narrative appears to represent structural patterns that are in fact transmedial but that we associate with certain specific media types. A literary text which focuses on visual and aural perception and frequently changes point of view is perceived as 
having filmic traits. A feature film that does not tell a linear story and instead appears to repeat and vary a single theme may seem 'musically structured' to the audience.

Transmediation and media representation are like two sides of the same coin. You cannot have one without the other. A film can transmediate the plot of a novel without drawing much attention to its source - but it is forced to include a minimum of media representation, directly or indirectly, such as a comment that the film is 'based on the novel of ...' Also, it is difficult, perhaps even impossible, to represent other media types without representing narratives, ideas and thoughts that are usually communicated via that particular kind of media type.

Combination, transmediation and media representation may look like three independent categories typical for different media types, but that is not the case: combination, transmediation and media representation are analytical approaches, and thus two or even three of them can be used to explore the very same media product. A film, for example, can be analysed from the perspective of combination: how moving images and auditory media types integrate. A film can be analysed as transmediation if we analyse the adaptation process that turns a novel into film. You can also study the role of different kinds of media that are represented in a film. And no matter which aspect you choose to focus on, you should at least consider how aspects of the other two support your analysis.

\section{References}

Elleström, L. 2014. Media transformation: The transfer of media characteristics among media. London: Palgrave Macmillan. 